\title{
Posterior translacrimal approach to the maxillary sinus
}

\author{
D DÖNMEZ ${ }^{1}$, E GIOTAKIS ${ }^{1}$, W G HOSEMANN ${ }^{3}$, T S KÜHNEL ${ }^{4}$, B HIRT $^{5}$, R K WEBER ${ }^{1,2}$ \\ ${ }^{1}$ Department of Otorhinolaryngology, Municipal Hospital of Karlsruhe, ${ }^{2} i$-SINUS International Sinus Institute, \\ Karlsruhe, ${ }^{3}$ Department of Otorhinolaryngology Head and Neck Surgery, University of Greifswald, ${ }^{4}$ Department \\ of Otorhinolaryngology Head and Neck Surgery, University of Regensburg, and ${ }^{5}$ Institute of Clinical Anatomy and \\ Cell Analysis, University of Tübingen, Germany
}

\begin{abstract}
Background: This study aimed to compare the view into the maxillary sinus using the posterior translacrimal approach compared with grade 3 antrostomy.

Methods: Grade 3 antrostomy followed by a posterior translacrimal approach was performed on four cadavers. The maximum intramaxillary view was documented endoscopically guided by electromagnetic navigation. Representative screenshots were evaluated in a blinded manner by three independent sinus surgeons. In addition, a prospective investigation of specific complications in the post-operative course of consecutive patients was performed.

Results: In the cadaver study, the posterior translacrimal approach provided a significantly better view into the maxillary sinus compared with grade 3 antrostomy. In the clinical study, only 1 out of 20 patients reported on a minor problem with lacrimal drainage at 6 months.

Conclusion: The posterior translacrimal approach to visualising the maxillary sinus should be considered a strong alternative to more radical techniques.
\end{abstract}

Key words: Surgical Endoscopy; Paranasal Sinus Diseases; Maxillary Sinus; Nasolacrimal Duct

\section{Introduction}

In endoscopic sinus surgery, the standard surgical approach to the maxillary sinus is performed through the middle nasal meatus. ${ }^{1,2}$ Specific interventions range from simply identifying the natural ostium to moderate or maximum antrostomy. ${ }^{2}$ The optimum size of antrostomy is determined by the nature, extent and location of the disease; it depends on the sinus pathology and may not always be clear cut. ${ }^{1,3,4}$ For example, in recurrent acute maxillary sinusitis, it is often recommended that the natural ostium not be touched. When the extent of maxillary disease requires manipulation inside the sinus, enlargement is required and must be achieved in a stepwise manner. ${ }^{2}$

The standard approach to the maxillary sinus can be classified into three grades. ${ }^{5,6}$ When planning the approach, it should also be considered that a surgically enlarged ostium tends to shrink by approximately 50 per cent. ${ }^{7,8}$

Despite using angled optics, variable sections of the maxillary sinus are often not visible and cannot be reached through the middle meatal antrostomy. ${ }^{9,10}$ In such cases, it is extremely difficult to be confident that lesions such as antrochoanal polyps, sticky mucous or recurrent polyps in chronic rhinosinusitis with nasal polyps have been completely removed.

When complete removal of recurrent polyposis, fungus balls, antrochoanal polyps or other benign processes is not possible with grade 3 (or maximal) middle meatal antrostomy, an alternative method for gaining access must be chosen, such as an inferior nasal meatus $^{11}$, prelacrimal ${ }^{12}$ or canine fossa trephine approach. $^{13}$

The posterior translacrimal approach has been recently described. ${ }^{2}$ This approach enlarges grade 3 middle meatal antrostomy anteriorly by removing the bony canal of the nasolacrimal duct medially, dorsally and laterally. Thus, the nasolacrimal duct can be mobilised anteriorly, offering a much better view into the zygomatic recess, the alveolar recess, the anterior wall of the maxillary sinus and the prelacrimal recess.

This study comprised two parts: (1) a cadaveric study comparing the view into the maxillary sinus achieved with the posterior translacrimal approach compared with grade 3 middle meatal antrostomy; 
and (2) a clinical study to identify specific short- and long-term complications that impair the lacrimal drainage system.

\section{Materials and methods}

\section{Surgery using the posterior translacrimal approach}

After performing standard grade 3 middle meatal antrostomy, a horizontal incision is made in the mucosa covering the frontal process of the maxilla and the lacrimal bone halfway between the inferior turbinate attachment and the plane of the maxillary roof. The mucosa is gently pushed inferiorly and superiorly to expose the bone. Subsequently, the medial parts of the frontal process of the maxilla are chiselled off tangentially to expose the soft tissues of the nasolacrimal duct. Using a bent freer elevator, the lacrimal bone and the posterior and lateral parts of the frontal process are mobilised and removed. The superior extent of bone removal is the level of the maxillary roof; the inferior extent is the level of the inferior turbinate attachment. The bony attachment of the inferior turbinate to the frontal process is removed. After removing its complete bony circumference, the membranous but intact nasolacrimal duct can be mobilised temporarily in an anterior direction, permitting improved access to the maxillary sinus (Figures 1 and 2).

\section{Study design}

The first part of the study included eight maxillary sinuses from four cadaveric specimens obtained through a donor programme guided by the Department of Clinical Anatomy and Cell Analysis at Eberhard Karls University, Tübingen, Germany. All donors gave written informed consent for the use of their bodies for research purposes. The donation programme complies with the Declaration of Helsinki and was approved by the Ethics Committee of the University of Tübingen (number 237/2007B01). All cadavers were free of

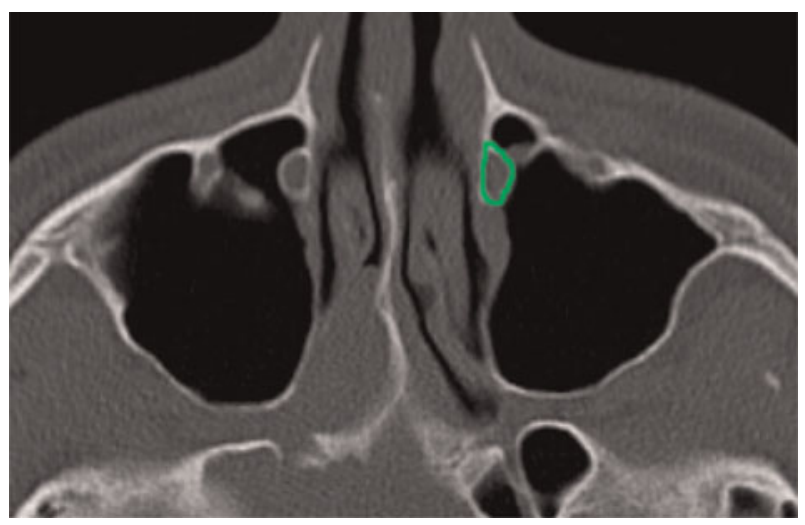

FIG. 1

Sinus computed tomography in the axial plane at the height of the maxillary sinus showing the posterior translacrimal approach with resection of the bone (green) medially, dorsally and laterally of the nasolacrimal duct on the left side. The bony canal of the nasolacrimal duct is clearly seen on the right side. recent trauma; they were fixed in 70 per cent ethanol and 30 per cent glycerol within 10-24 hours of death.

Grade 3 middle meatal antrostomy was performed using a $45^{\circ}$ endoscope and microsurgical instruments (Karl Storz, Tuttlingen, Germany) with the goal of achieving the maximum view into the maxillary sinus. Results were documented by high-definition video endoscopy (AIDA, Karl Storz) using an electromagnetic navigation system (pointer) and a curved suction device to pinpoint and localise the four

(a)

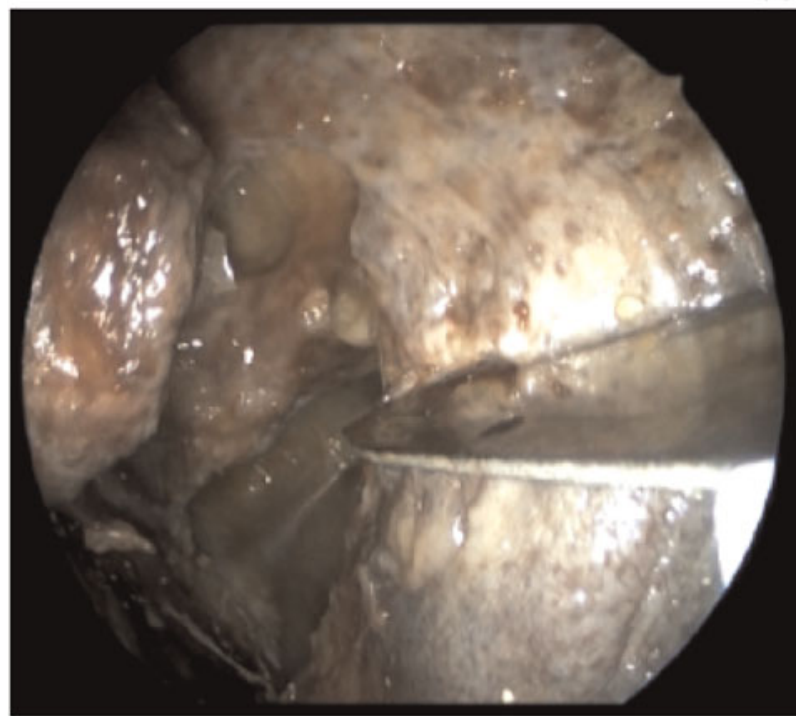

(b)

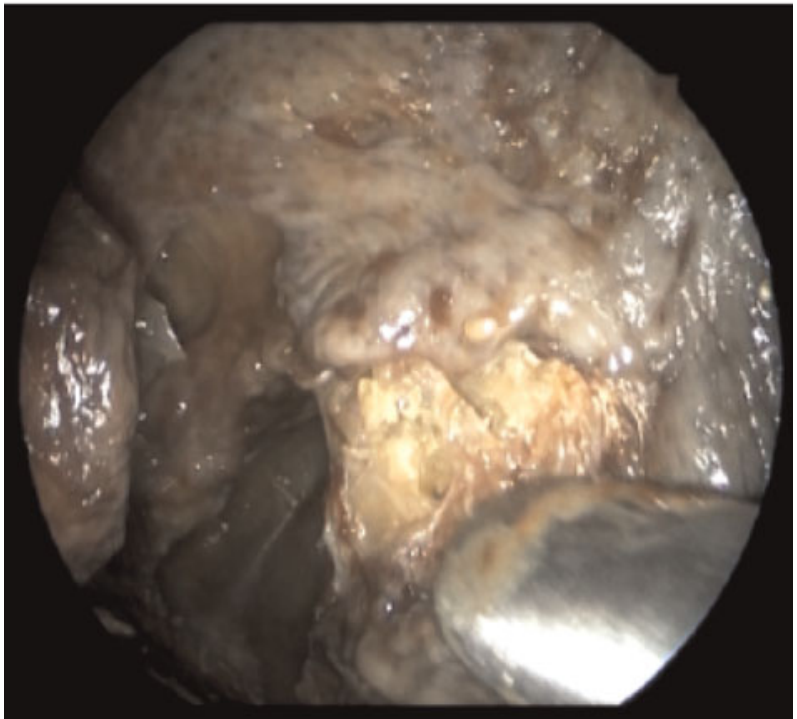

FIG. 2

Endoscopic views of the surgical technique used for cadaver dissection with the posterior translacrimal approach (left side, $45^{\circ}$ endoscope) showing (a) a horizontal incision at the lateral nasal wall a few millimetres above the attachment of the inferior turbinate; (b) elevation of mucosal flaps superiorly and inferiorly with exposure of the frontal process of the maxilla; (c) exposure of the nasolacrimal duct after removing the frontal process of the maxilla with a chisel; (d) mobilisation of the nasolacrimal duct to expose the posterior and lateral part of the bony canal, allowing subsequent removal of this bone; and (e) mobilisation and removal of the bone in the transition zone to the inferior turbinate attachment. 
(c)

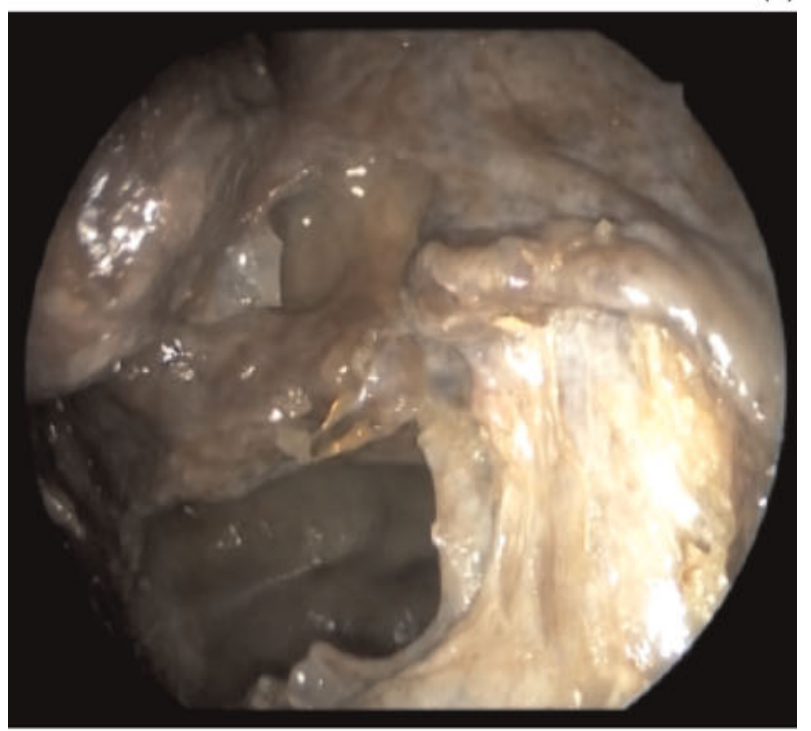

(d)

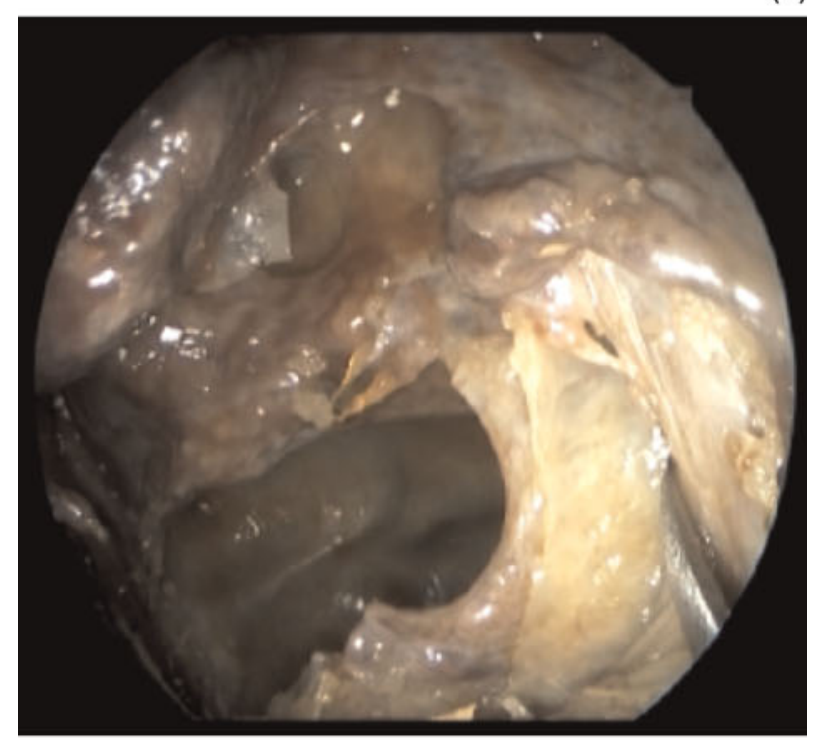

(e)

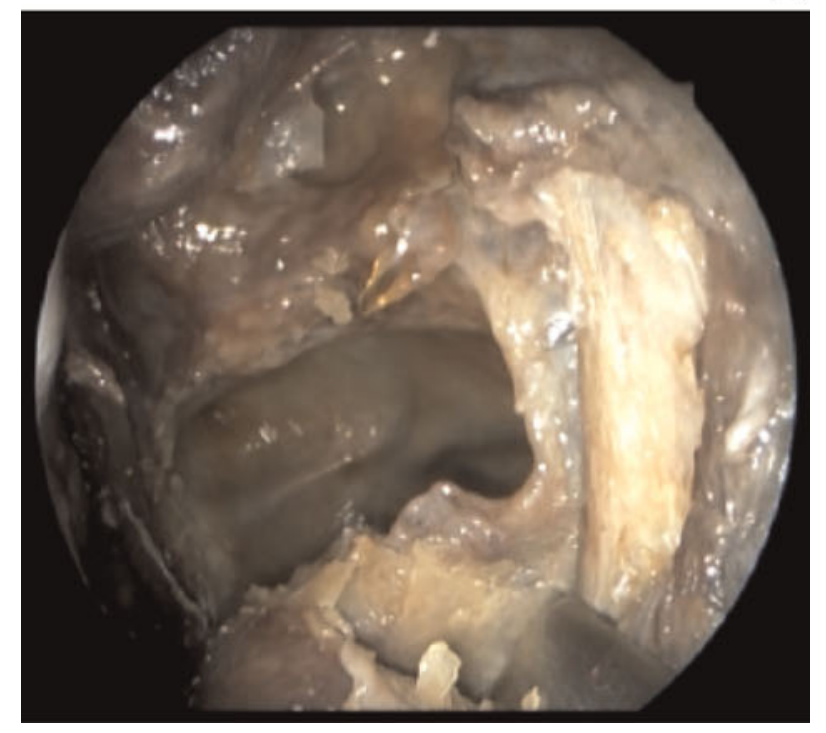

FIG. 2

Continued. following regions of interest: the zygomatic recess; the alveolar recess; the anterior wall; and the prelacrimal recess of the maxillary sinus. The process was evaluated by screenshots taken through the ipsilateral nostril. Next, the existing antrostomy was enlarged using a posterior translacrimal approach (as described above) using identical instruments and endoscopes. Results were documented as described above.

Eight representative screenshots from the endoscopy navigation system were taken for each intervention and four different regions of interest for each specimen, with the type of antrostomy concealed. Three independent experienced sinus surgeons blinded to the type of intervention evaluated the complete set of screenshots for the quality of endoscopy control for the selected maxillary sinus regions using the following scoring system: $0=$ no control possible; $1=$ up to 50 per cent visibility; $2=$ more than 50 per cent visibility, and $3=100$ per cent visibility.

In the second part of the study, the post-operative course of 14 consecutive patients who underwent maxillary sinus surgery with a posterior translacrimal approach was prospectively analysed for possible impairment of lacrimal transport or irritation of the lacrimal drainage pathway. The enrollment period was August 2015 to March 2016. Informed consent was obtained from all participants and ethics committee approval was obtained from the State Medical Chamber of Baden-Württemberg and complied with the 1964 Helsinki declaration and its later amendments or comparable ethical standards.

The presence of the subjective symptom of decreased lacrimal transport was scored using the following scale: $0=$ no deterioration; $1=$ mild deterioration; and $2=$ severe deterioration. Passive lacrimal transport was assessed by irrigating the lacrimal system via the inferior lacrimal punctum using a 2-ml syringe and lacrimal irrigation cannula and scored as: $0=$ irrigation without resistance; $1=$ irrigation with increased resistance; or 2 =irrigation not possible. Active lacrimal transport was assessed by applying a drop of fluorescein solution into the conjunctival sac and scored as: $0=$ normal transport (within 2 minutes); $1=$ delayed transport time $(3-5$ minutes $)$; or $2=$ no active transport. Early and delayed impairment in lacrimal transport were scored at post-operative days two to four; weeks one, two and four; and months three and six.

\section{Statistical analysis}

Three datasets (one from each of the three investigators) were analysed, each comprising 64 images captured from 4 regions of each maxillary sinus using both surgical techniques $(4 \times 8 \times 2=64)$. The null hypothesis was that there was no difference between the two surgical methods in terms of visibility of the maxillary sinus for four representative regions of interest inside the sinuses. There was no significant difference in the quality of the endoscopic view into the 
maxillary sinus between the two different types of antrostomy.

The means and standard deviations of the scores were calculated for both surgical methods and the distribution of values was compared between the two interventions. The results were revised using one- and two-sided Kolmogorov-Smirnov tests, two-sample $t$-tests and Mann-Whitney U-tests. Finally, threefold analysis of variance was performed to evaluate the effect of the following variables: investigator; surgical method; and maxillary sinus region. The dependent variable, $Y$, reflecting the surgeon's individual assessment of the view, was set at a value between 1 (very poor) and 4 (excellent).

\section{Results}

\section{Cadaveric study}

Comparison of means and standard deviations of scores. The mean score was larger for the posterior translacrimal approach (method two; mean 2.072917) than for antrostomy type 3 (method 1 ; mean 1.333333), indicating that the posterior translacrimal approach gave a better surgical view into the maxillary sinus. The standard deviation was similar for both datasets (1.092237 for the posterior translacrimal approach and 0.8854947 for antrostomy type 3 ), indicating that comparing the means was a reasonable analysis.
Comparison of the distribution and dominance parameters. Distribution and dominance are statistical parameters for describing the similarities between datasets. In this analysis, differences in these parameters indicate differences in the endoscopic view. All statistical tests showed that a significantly better view into the maxillary sinus was achieved using the posterior translacrimal approach compared with antrostomy type 3 (Table I).

Analysis of variance. Correlations between the command variable, $y$ (i.e. endoscopic view into the maxillary sinus), and potential interactions were determined using the following equation:

$$
\mathrm{y}=\alpha+\beta_{1}+\beta_{2}+\beta_{3}+\gamma_{12}+\gamma_{13}+\gamma_{23}+\delta_{123}+\varepsilon,
$$

where $\alpha=$ absolute term; $\beta_{1}=$ effect of person (three values); $\beta_{2}=$ effect of method (two values); $\beta_{3}=$ effect of region (four values); $\gamma_{12}, \gamma_{13}, \gamma_{23}, \delta_{123}=$ pairwise and triple interactions; and $\varepsilon=$ idiosyncratic values.

Analysis of variance showed that the effects of method and region were highly significant (Table II). Interactions $\left(\gamma_{12}, \gamma_{13}, \gamma_{23}, \gamma_{123}\right)$ were not significant; hence, the effects were additive and can be considered as independent from one another.

In summary, the descriptive statistical analysis showed higher mean values and smaller deviations for the posterior translacrimal approach, providing

\begin{tabular}{|c|c|c|c|c|}
\hline \multicolumn{5}{|c|}{$\begin{array}{c}\text { TABLE I } \\
\text { TESTS FOR VARIANCE OF DISTRIBUTION }\end{array}$} \\
\hline Test & Hypothesis & df & Test statistics & $p$ value \\
\hline Two-sample Kolmogorov-Smirnov test & $\begin{array}{l}\mathrm{H}_{0}: \text { distribution is similar } \\
\mathrm{H}_{1} \text { : distribution is different }\end{array}$ & - & $D=0.35417$ & $1.179 \times 10^{-5}$ \\
\hline Two-sample $t$-test & $\begin{array}{l}\mathrm{H}_{0} \text { : mean values are the same } \\
\mathrm{H}_{1} \text { : mean values are different }\end{array}$ & 95 & $t=-9.2352$ & $7.129 \times 10^{-15}$ \\
\hline $\begin{array}{l}\text { Mann-Whitney U-Test with adjustment } \\
\text { of consistency and steadiness }\end{array}$ & $\begin{array}{l}\mathrm{H}_{0} \text { : distribution is the same } \\
\mathrm{H}_{1} \text { : distribution is shifted }\end{array}$ & - & $V=74$ & $7.772 \times 10^{-11}$ \\
\hline $\begin{array}{l}\text { Two-sample Kolmogorov-Smirnov test } \\
\text { for stochastic dominance }\end{array}$ & $\begin{array}{l}\mathrm{H}_{0} \text { : distribution is the same } \\
\mathrm{H}_{1} \text { : distribution } 2 \text { is dominated } \\
\text { by distribution } 1\end{array}$ & - & $D^{+}=0.35417$ & $5.893 \times 10^{-6}$ \\
\hline
\end{tabular}

Df $=$ degrees of freedom; $\mathrm{H}_{0}=$ null hypothesis; $\mathrm{H}_{1}=$ alternative hypothesis

\begin{tabular}{lccccc}
\multicolumn{7}{c}{ TABLE II } \\
& \multicolumn{7}{c}{ THREEFOLD ANALYSIS OF VARIANCE } \\
\hline Parameter & df & Sum sq & Mean sq & $F$ value & $p$ value \\
\hline Person $\beta_{1}$ & 2 & 0.41 & 0.2 & 0.465 & 0.629 \\
Method $\beta_{2}$ & 1 & 26.26 & 26.26 & 60.114 & $8.18 \times 10^{-13 *}$ \\
Region $\beta_{3}$ & 3 & 108.77 & 36.26 & 83.01 & $<2.00 \times 10^{-16 *}$ \\
Person: method $\gamma_{12}$ & 2 & 1.26 & 0.63 & 1.443 & 0.239 \\
Person: region $\gamma_{13}$ & 6 & 0.97 & 0.16 & 0.37 & 0.897 \\
Method: region $\gamma_{23}$ & 3 & 2.18 & 0.73 & 0.666 & 0.176 \\
Person: method: region $\delta_{123}$ & 6 & 0.86 & 0.14 & 0.43 \\
Residuals $\varepsilon$ & 168 & 73.37 & & & \\
\hline
\end{tabular}

${ }^{*} p<0.001 . \mathrm{Df}=$ degree of freedom; $\mathrm{Sq}=$ square 


\begin{tabular}{|c|c|c|c|c|c|c|c|c|}
\hline & $\mathrm{IM}$ & TR & NSPO & $\begin{array}{l}\text { ABLE } \\
\text { T: SL }\end{array}$ & & E S & IPTO & \\
\hline Score & D 2 & D 3 & D 4 & W 1 & W 2 & W 4 & M 3 & M 6 \\
\hline 0 & 18 & 24 & 21 & 22 & 24 & 22 & 21 & 19 \\
\hline 1 & 4 & 0 & 2 & 2 & 0 & 1 & 2 & 1 \\
\hline 2 & 0 & 0 & 0 & 0 & 0 & 0 & 0 & 0 \\
\hline
\end{tabular}

Data are $n$ values (number of patients). *After surgery with the posterior translacrimal approach. Score: $0=$ no deterioration; $1=$ mild deterioration; $2=$ severe deterioration. $\mathrm{D}=$ post-operative day, $\mathrm{W}=$ post-operative week, $\mathrm{M}=$ post-operative month

supportive evidence that this approach enables a better view into the maxillary sinus compared with grade 3 antrostomy. The distribution of values was also significantly different between surgical approaches, favouring the posterior translacrimal approach. The three-way analysis of variance yielded similar results.

\section{Clinical study: analysis of the lacrimal transport}

A total of 14 patients underwent 24 posterior translacrimal operations. Surgical indications were antrochoanal polyp $(n=1)$, fungus ball $(n=2)$, chronic rhinosinusitis with nasal polyps ( $n=15$, with 8 revision cases), chronic rhinosinusitis without nasal polyps $(n=2$, with 2 revision cases) and recurrent acute maxillary sinusitis ( $n=4$, with 2 revision cases).

Four patients did not attend the final examination at six months because one had undergone cochlear implantation in the interim and three could not take time off work to attend. At earlier investigations, all had findings of normal active lacrimal transport and irrigation and reported no symptoms.

In all, 19 of 20 patients had no subjective deterioration of lacrimal transport. Only one patient had the slight complaint of mucous secretion and air coming out of the lacrimal punctum during nose blowing (Table III); this patient had normal passive and active lacrimal transport at examination. Five patients experienced increased resistance during irrigation of the lacrimal system (Table IV); none of these reported any lacrimal symptoms. Assessment of active lacrimal transport with fluorescein showed normal findings in all patients (Table V).

In summary, no clinically relevant impairment of lacrimal transport due to lacrimal stenosis was found

\begin{tabular}{lrrrrrrrr}
\multicolumn{7}{c}{ TABLE IV } \\
\multicolumn{7}{c}{ PASSIVE LACRIMAL TRANSPORT* } \\
\hline Score & D 2 & D 3 & D 4 & W 1 & W 2 & W 4 & M 3 & M 6 \\
\hline 0 & 10 & 18 & 22 & 19 & 24 & 19 & 21 & 15 \\
1 & 12 & 6 & 1 & 5 & 0 & 4 & 2 & 5 \\
2 & 0 & 0 & 0 & 0 & 0 & 0 & 0 & 0 \\
\hline
\end{tabular}

Data are $n$ values (number of patients). *After surgery with the posterior translacrimal approach. Score: $0=$ irrigation without resistance; 1 = irrigation with increased resistance; $2=$ irrigation not possible. $\mathrm{D}=$ post-operative day, $\mathrm{W}=$ post-operative week, $\mathrm{M}=$ post-operative month

\begin{tabular}{lrrrrrrrr}
\multicolumn{1}{c}{ TABLE V } \\
\multicolumn{7}{c}{ ACTIVE LACRIMAL TRANSPORT* } \\
\hline Score & D 2 & D 3 & D 4 & W 1 & W 2 & W 4 & M 3 & M 6 \\
\hline 0 & 18 & 24 & 22 & 24 & 24 & 20 & 21 & 20 \\
1 & 4 & 0 & 1 & 0 & 0 & 3 & 2 & 0 \\
2 & 0 & 0 & 0 & 0 & 0 & 0 & 0 & 0 \\
\hline
\end{tabular}

Data are $n$ values (number of patients). ${ }^{*}$ After surgery with the posterior translacrimal approach. Score: $0=$ normal transport (within 2 minutes); $1=$ delayed transport time (3-5 minutes); $2=$ no active transport. $\mathrm{D}=$ post-operative day, $\mathrm{W}=$ post-operative week, $\mathrm{M}=$ post-operative month

at six months. There was no evidence that patients with worse results did not attend later follow-up investigations.

\section{Discussion}

Although middle meatal antrostomy is the current standard surgical approach used to access the maxillary sinus and is suitable for most diseases, ${ }^{1,3,4}$ some pathological conditions require precise, complete removal of the specific lesion under endoscopic control to minimise the likelihood of recurrence. Examples include antrochoanal polyps, maxillary sinus cysts, ${ }^{14}$ recurrent polyposis and sticky secretions, fungus balls, and benign tumours. ${ }^{2}$

The use of endoscopes with different angles of view has improved visualisation and precision in maxillary sinus surgery. Nevertheless, handling endoscopes with uncommon angulations (i.e. of greater than $45^{\circ}$ ) can be extremely difficult, even for experienced sinus surgeons.

Sometimes a disease process in the maxillary sinus cannot be visualised completely even when using endoscopes with large angles of view and after grade 3 middle meatal antrostomy. Such cases require supplementary approaches through the inferior nasal meatus such as the prelacrimal, medial maxillectomy or canine fossa trephine approach. ${ }^{2,11-13}$ Compared with the posterior translacrimal approach, these options require more pronounced manipulation, which can be associated with additional morbidity such as damage to branches of the infraorbital nerve or nasolacrimal duct and Hasner's valve, as well as disturbance of the maxillary sinus ciliary physiology by inferior antrostomy.

The aim of the posterior translacrimal approach is to overcome a limited endoscopic view into the maxillary sinus by anterior mobilisation of the soft tissues of the nasolacrimal duct freed from its bony circumference, comprising part of the frontal process of the maxilla, most sections of the lacrimal bone and a bony triangle between the inferior turbinate, medial wall of the maxillary sinus and nasolacrimal duct (Figures 1 and 2).

This prospective study demonstrated that the posterior translacrimal approach provides a much better view into the zygomatic recess, alveolar recess, prelacrimal recess and anterior wall of the maxillary sinus (Figures 3 and 4, Table II). 


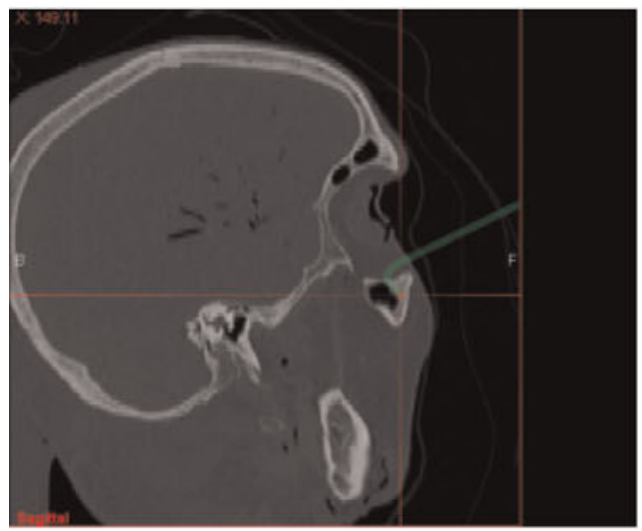

(a)
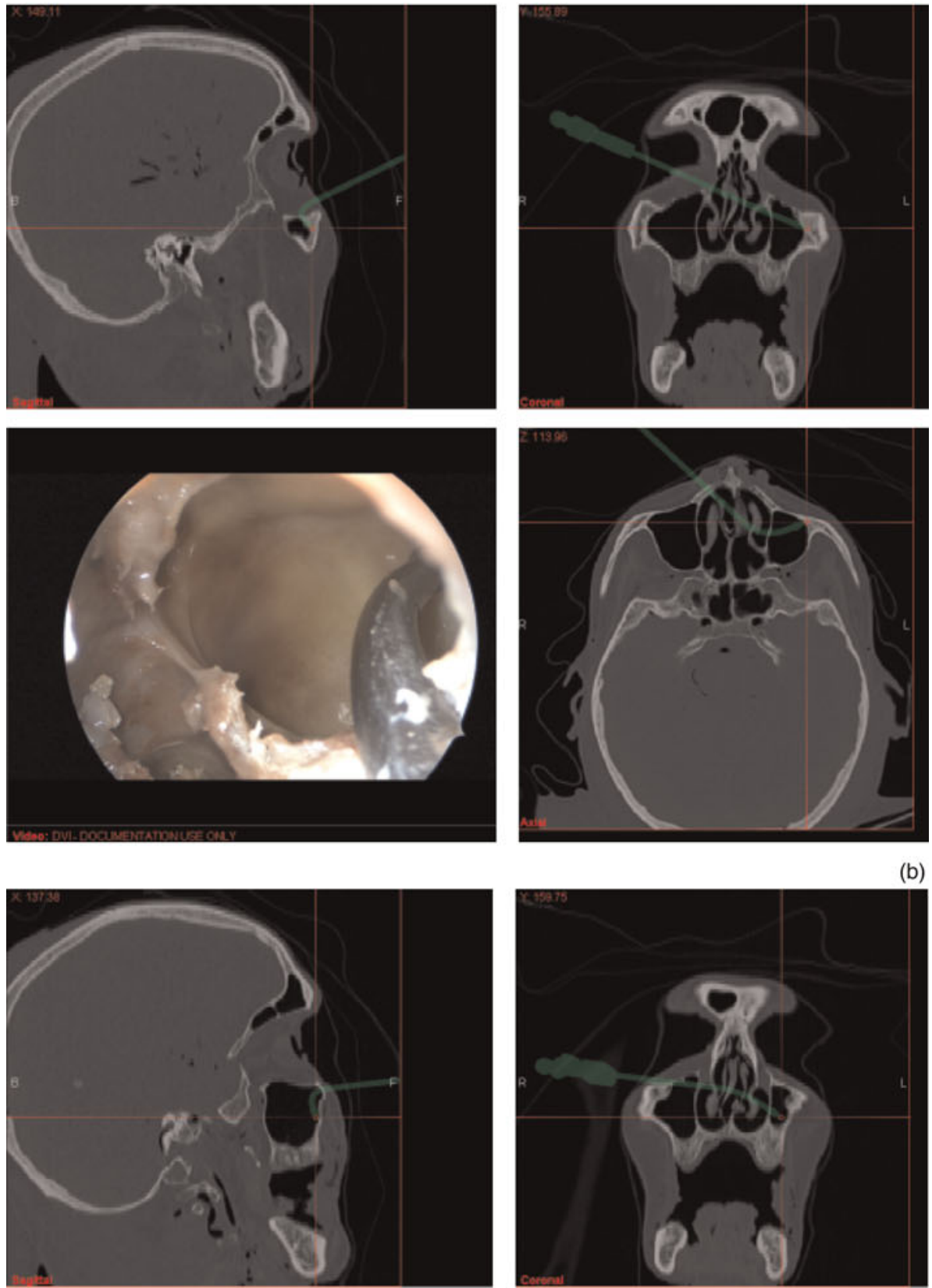

(b)
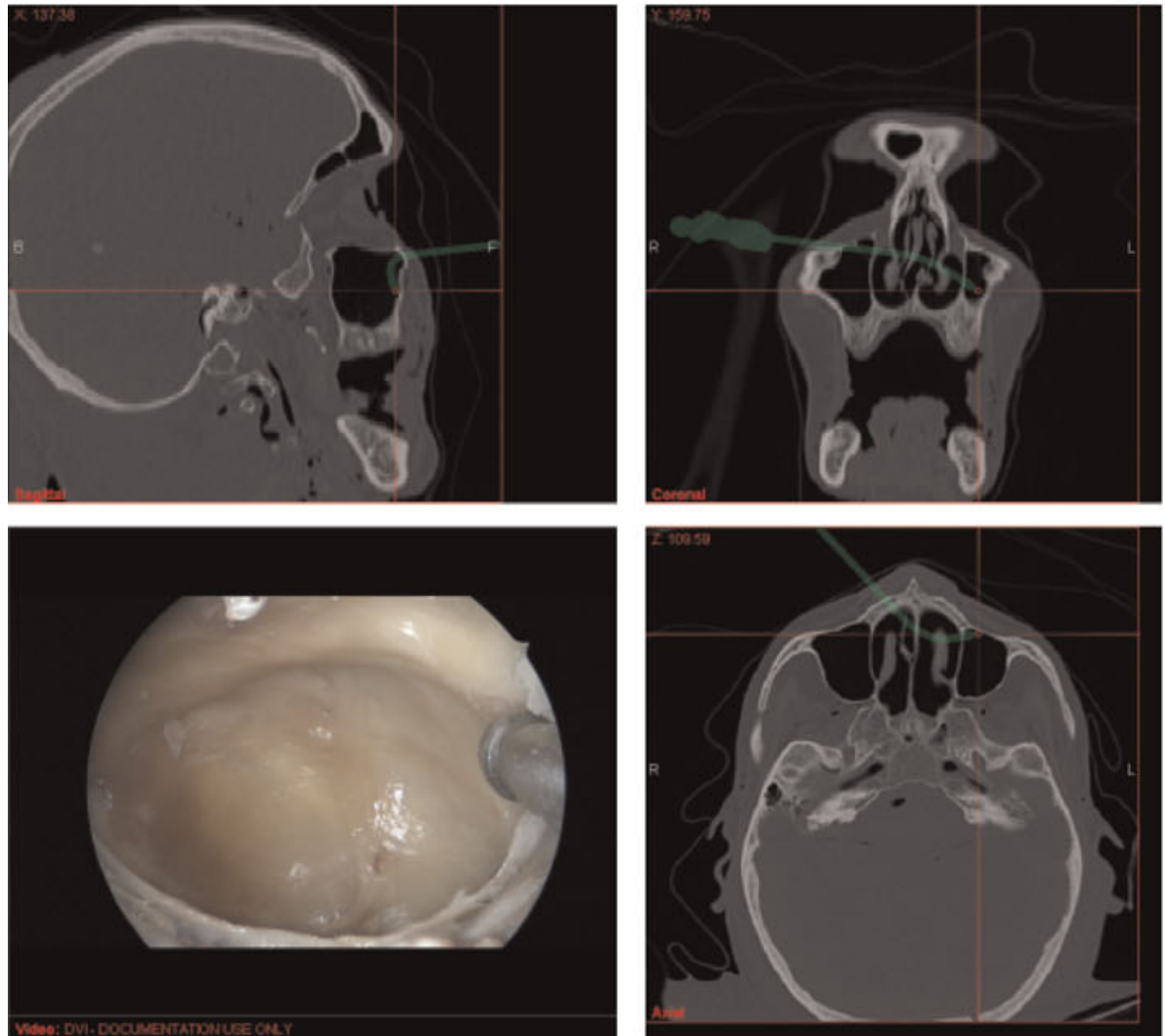

FIG. 3

Screen shots of the zygomatic recess or maxillary sinus obtained by grade 3 antrostomy and the posterior translacrimal approach: top left, sinus computed tomography in the sagittal plane; top right, sinus computed tomography in the coronal plane; bottom left, corresponding endoscopic view using a $45^{\circ}$ optic; and bottom right, sinus computed tomography in the axial plane. (a) After grade 3 middle meatal antrostomy, the zygomatic recess is incompletely exposed by the $45^{\circ}$ endoscope. (b) After mobilising the nasolacrimal duct with the posterior translacrimal approach, the zygomatic recess can be completely controlled by the endoscope (more than 50 per cent of the anterior wall of the maxillary sinus is visible). 

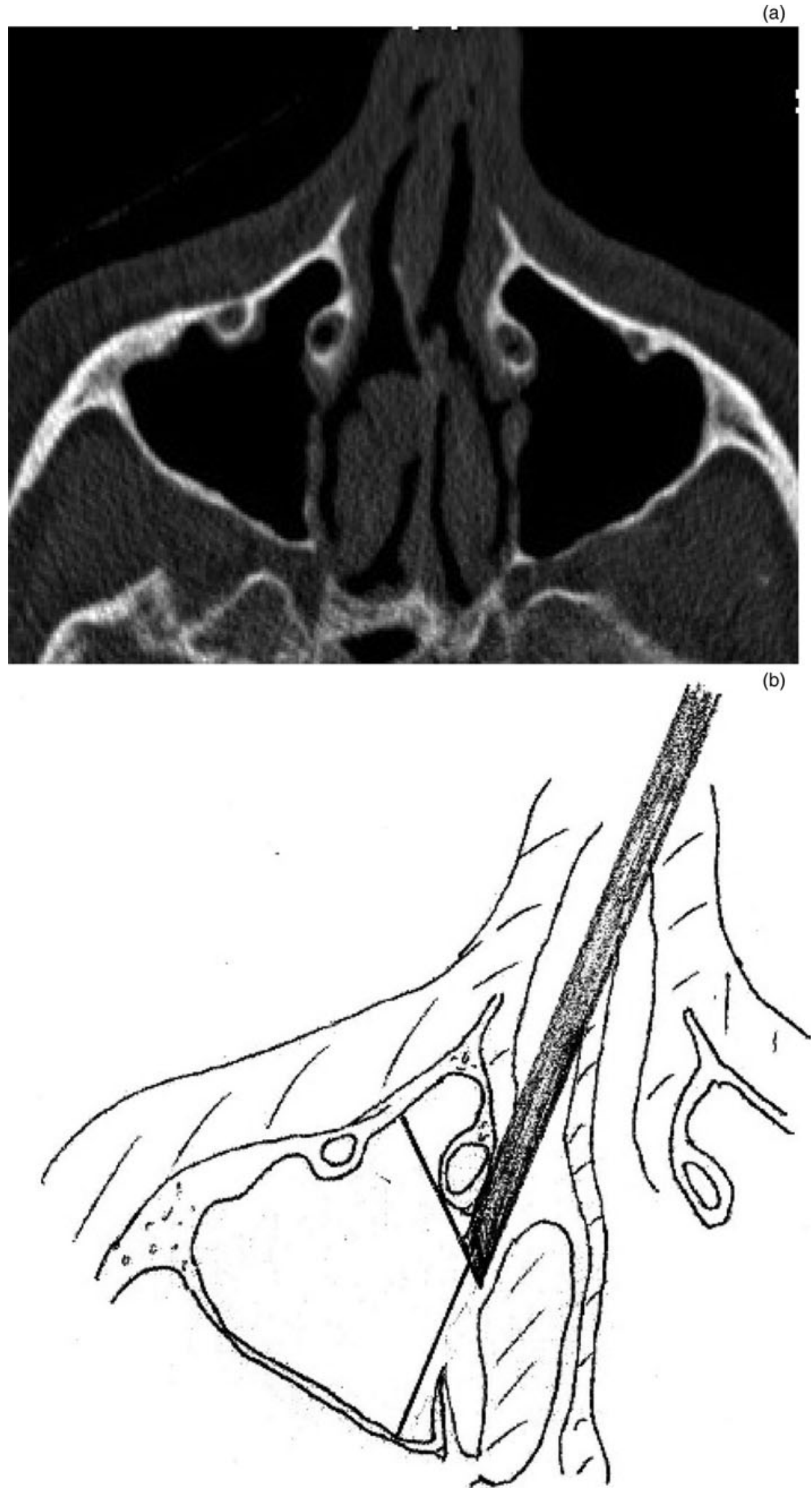

FIG. 4

Sinus computed tomography and schematic drawings showing the view into the maxillary sinus before and after surgery with the posterior translacrimal approach. (a) Sinus computed tomography in the axial plane. (b) Best endoscopic view into the maxillary sinus with a $45^{\circ}$ wide-angle optic $\left(130^{\circ}\right.$ viewing angle) after middle meatal antrostomy grade 3. (c) Bone resection for the posterior translacrimal approach and direction of mobilisation of nasolacrimal duct (indicated by black colour and arrow). (d) The posterior translacrimal approach provides a significantly better endoscopic view into the maxillary sinus. 


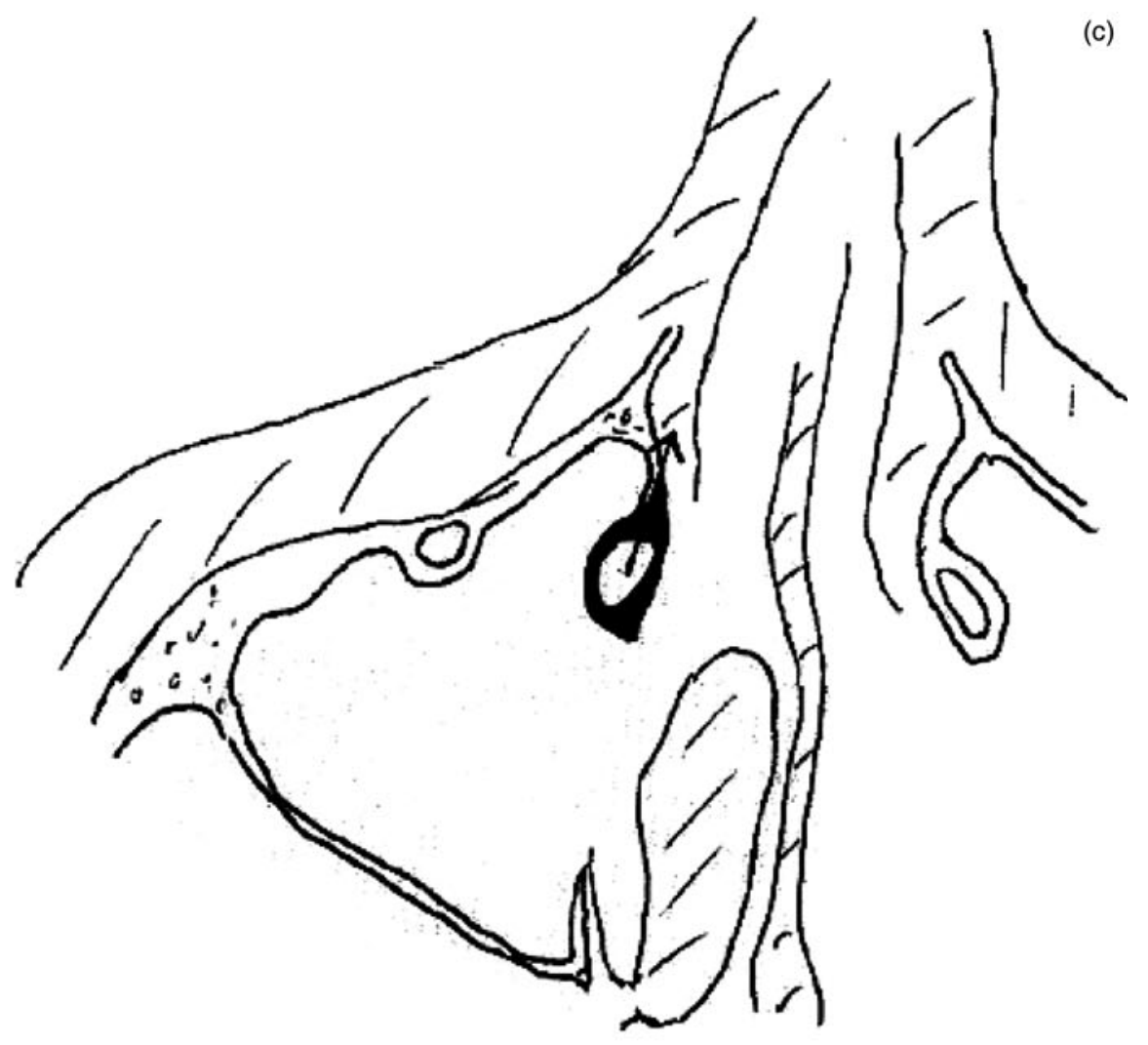

(d)

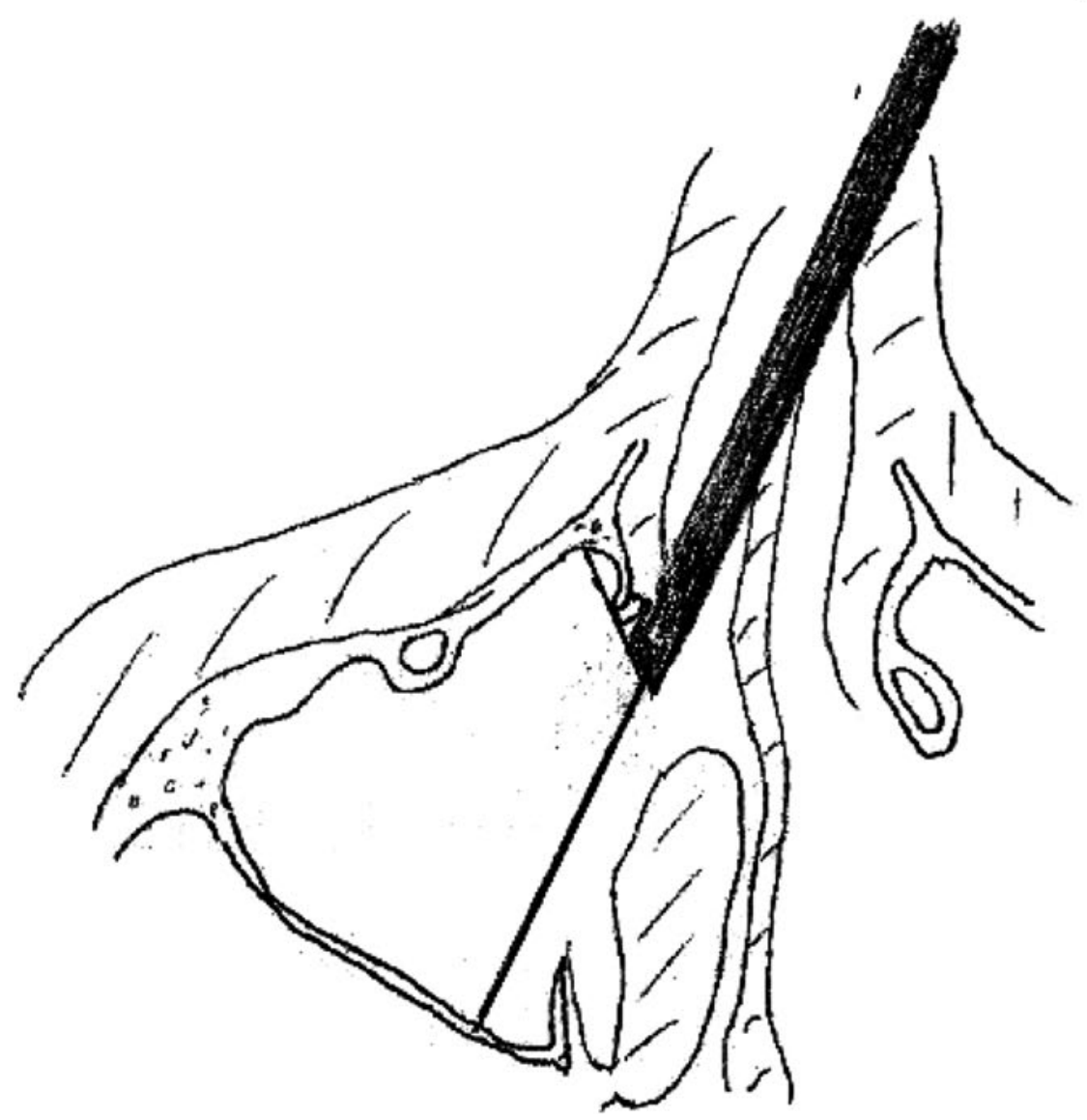

FIG. 4

Continued. 
This new surgical approach allows complete removal of the pathological lesion with no evidence of additional morbidity. In addition, the operative time is shortened and there is no risk of major bleeding (because no major blood vessels are present in the surgical area).

The prospective clinical evaluation also demonstrated that no lacrimal stenosis occurs due to mobilising the nasolacrimal duct and removing the surrounding bone. The six-month follow-up data represent long-term outcomes. In only one patient, injury to the nasolacrimal duct caused air and mucosal secretion to be expelled during nose blowing. There were no other complications.

- Variable areas of the maxillary sinus are often not visible and cannot be reached via middle meatal antrostomy

- The inferior nasal meatus, prelacrimal and canine fossa trephine approaches are alternative access routes for middle meatal antrostomy

- The posterior translacrimal approach aims to overcome a limited endoscopic view by anterior mobilisation of soft tissues of the nasolacrimal duct

- This approach provides significantly better intramaxillary visualisation compared with grade 3 antrostomy

- All of the alternative techniques require more additional manipulation, which is associated with additional morbidity

- The posterior translacrimal approach is thus a good alternative to more radical techniques

A limitation of this anatomical study was the small number of dissections and clinical patients included. Despite this, there was a clear, significant difference between surgical approaches. A second limitation is that assessment of the endoscopic view was semi-quantitative when electromagnetic navigation was used to verify the position of the probe (i.e. a measuring tool was not used). However, despite the low number of patients, the risk of lacrimal stenosis is obviously very low.

\section{Conclusion}

The posterior translacrimal maxillary sinus approach provides significantly better visualisation into the maxillary sinus with little extra morbidity compared with grade 3 maxillary sinus antrostomy, Therefore, the posterior translacrimal approach is a good alternative to more radical techniques for treating maxillary sinus pathology. Prospective clinical studies are needed to determine whether the posterior translacrimal approach is clinically superior to current approaches.

\section{Acknowledgements}

The authors thank Dr M Höchstötter, Karlsruhe Institute of Technology, for performing the statistical analysis. This study was funded by the Institute of Clinical Anatomy and Tissue Engineering, University of Tübingen.

\section{References}

1 Kennedy DW, Adappa ND. Endoscopic maxillary antrostomy: not just a simple procedure. Laryngoscope 2011;121:2142-5

2 Weber RK, Hosemann W. Comprehensive review on endonasal endoscopic sinus surgery. GMS Curr Top Otorhinolaryngol Head Neck Surg 2015;14:1-108

3 Stammberger H. Comments on the contribution by U. Goede. Maxillary sinus fenestration via the middle vs. lower nasal passage [in German]. HNO 1998;46:95-101

4 Konstantinidis I, Constantinidis J. Medial maxillectomy in recalcitrant sinusitis: when, why and how? Curr Opin Otolaryngol Head Neck Surg 2014;22:68-74

5 Simmen D, Jones NS. Manual of endoscopic sinus and skull base surgery, 2nd edn. Stuttgart, New York: Thieme, 2013; 8:91-106

6 Wormald PJ. Endoscopic sinus surgery, 3rd edn. Stuttgart, New York: Thieme, 2013;5:28-44

7 Albu S, Tomescu E. Small and large middle meatus antrostomies in the treatment of chronic maxillary sinusitis. Otolaryngol Head Neck Surg 2004;131:542-7

8 Ngoc Ha T, Valentine R, Moratti S, Robinson S, Hanton L, Wormald PJ. A blinded randomized controlled trial evaluating the efficacy of chitosan gel on ostial stenosis following endoscopic sinus surgery. Int Forum Allergy Rhinol 2013;3:573-80

9 Hosemann W, Scotti O, Bentzien S. Evaluation of telescopes and forceps for endoscopic transnasal surgery on the maxillary sinus. Am J Rhinol 2003;17:311-16

10 Robey A, O'Brien EK, Leopold DA. Assessing current technical limitations in the small-hole endoscopic approach to the maxillary sinus. Am J Rhinol Allergy 2010;24:396-401

11 Albu S, Gocea A, Necula S. Simultaneous inferior and middle meatus antrostomies in the treatment of the severely diseased maxillary sinus. Am J Rhinol Allergy 2011;25:e80-e85

12 Zhou B, Han DM, Cui SJ, Huang Q, Wei YX, Liu HC et al. Endoscopic nasal lateral wall dissection approach to maxillary sinus [in Chinese]. Zhonghua Er Bi Yan Hou Tou Jing Wai Ke Za Zhi 2007;42:743-8

13 Sathananthar S, Nagaonkar S, Paleri V, Le T, Robinson S, Wormald PJ. Canine fossa puncture and clearance of the maxillary sinus for the severely diseased maxillary sinus. Laryngoscope 2005;115:1026-9

14 Giotakis EI, Weber RK. Cysts of the maxillary sinus: a literature review. Int Forum Allergy Rhinol 2013;3:766-71

Address for correspondence:

Dr D Dönmez,

Department of Otorhinolaryngology,

Municipal Hospital of Karlsruhe,

Moltkestraße 90

D- 76133 Karlsruhe, Germany

Fax: +497219742509

E-mail: duygu.doenmez@klinikum-karlsruhe.de

Dr D Dönmez takes responsibility for the integrity of the content of the paper

Competing interests: None declared 